\title{
Study on Separation of Ship Resistance Components
}

\author{
By Eiichi Baba*, Member
}

\begin{abstract}
Summary
This paper describes theoretical and experimental studies on the separation of ship total resistance into its components with physical meanings, namely viscous resistance and wave resistance. The theoretical analysis gives the asymptotic formulas of both components with linear approximation. The experimental data are analysed by use of the asymptotic formulas. The sum of both components is in good agreement with the total resistance derived by dynamometer. This study also indicates that the interaction term of these components can be neglected as a higher order quantity.
\end{abstract}

\section{Introduction}

In 1951 Tulin [1] suggested the possibility of separation of ship total resistance into wave resistance and viscous resistance by means of the wake survey. In 1962 Eggers [2] presented a theoretical study on methods of calculation of wave resistance from measured wave patterns. Since then, several investigators have tried to obtain more refined techniques of wake survey and wave analysis. In recent years Sharma [3] developed a method of wave analysis based on the measurement of waves along a longitudinal cut. Landweber and others [4] also developed a technique of wake survey and discussed the variation of viscous resistance as it relates with Froude number.

Unfortunately, however, only a few examples have been given in which both measurements of viscous resistance and wave resistance were used to determine the total resistance [5], [6]. Recently, in Mitsubishi Experimental Tank, extensive tests have been made on a variety of ship forms including Wigley's mathematical form and geosims of tankers to measure each resistance component separately. New method of theoretical analysis, based on a linear approximation of velocity field, have also been developed on the separation of the resistance components, leading to asymptotic formula for each component. The sum of the viscous resistance and the wave resistance analysed by this theory was found to be in good agreement with the total resistance measured by dynamometer. In the following is presented outline of those recent investigations.

\section{Theoretical study on separation of total resistance of ship}

\subsection{General expressions of ship resistance}

Applying the conservation principle of mass and momentum to viscous incompressible fluid in steady motion, following basic equations can be written

$$
\begin{aligned}
& \nabla \boldsymbol{q}=0 \\
& \rho(\boldsymbol{q} \nabla) \boldsymbol{q}=\nabla \Phi+\rho \boldsymbol{F}
\end{aligned}
$$

where $\boldsymbol{q}$ is the velocity vector, $\rho$ the density of fluid, $\boldsymbol{F}$ the external force per unit mass such as gravity, and $\Phi$ the stress tensor. The stress on the surface of fluid is given by

$$
\boldsymbol{n} \Phi=-p \boldsymbol{n}+\mu[2(\boldsymbol{n} \boldsymbol{\nabla}) \boldsymbol{q}+\boldsymbol{n} \times \boldsymbol{\omega}]
$$

where $p$ is the pressure, $\mu$ the coefficient of viscosity, $\boldsymbol{n}$ the unit outward normal, and $\boldsymbol{\omega}=\boldsymbol{\nabla} \times \boldsymbol{q}$ the vorticity [7]. The second term of the right hand side of (3) is the stress due to friction.

* Experimental Tank, Nagasaki Technical Institute, Mitsubishi Heavy Industries, Ltd. 
In addition to the basic equations (1) and (2) the boundary conditions should be considered. On the surface of the fluid the kinematical boundary condition should be satisfied, i.e. $\boldsymbol{n} \boldsymbol{q}=0$. In the case of viscous fluid the following dynamical boundary conditions should be satisfied, viz., on the surface of a ship,

$$
q=0
$$

on the free surface of the fluid neglecting surface tension,

$$
\boldsymbol{n} \Phi=0
$$

If $\mu=0$, the condition ( $4 \mathrm{~b}$ ) is identical with the condition of

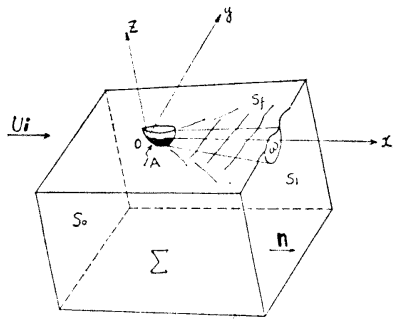

Fig. 1. Coordinate system and control surfaces constant pressure on the free surface of the inviscid fluid.

Now consider a ship floating in a uniform stream $\boldsymbol{U}=U \boldsymbol{i}$. Fig. 1 shows the coordinate system for the ship and other symbols to be used in the following discussion. $\mathrm{x}$-axis coincides with the direction of uniform flow with its origin at the F.P. of the ship, y-axis being horizontal and normal to $x$-axis, $z$-axis directing vertically upwards. $\Sigma$ the surface of the parallelpiped surrounding the ship, of which $S_{f}$ is the free surface, $S_{0}$ and $S_{1}$ are perpendicular to the uniform stream. $A$ is the surface of the ship, $\omega$ is the sectional area of the viscous wake.

By Gauss's theorem the integration of equation (2) over the volume $V$ surrounded by $\Sigma$ and $A$ gives the following equation, since $n q=0$ on $A$,

$$
\int_{A} \boldsymbol{n} \Phi d s=\int_{V} \rho \boldsymbol{F} d \tau+\int_{\Sigma}[\boldsymbol{n} \Phi-\rho(\boldsymbol{n} \boldsymbol{q}) \boldsymbol{q}] d s
$$

where $d \tau$ is the volume element. The integral on $A$ shows the resultant force acting on the ship. because $n \Phi$ is the stress on the surface of the ship. The relation (5) indicates that there are two ways of expressing ship resistance, i.e. the one by the integration of stress on the surface of the ship and the other by the integration of stress and rate of momentum on the control surfaces at a distance from the ship, since there is no contribution from the free surface of the fluid because of the boundary conditions $\boldsymbol{n} \Phi=0$ and $\boldsymbol{n} \boldsymbol{q}=\mathbf{0}$.

Consider first the resistance which is expressed by integration of stress on the surface of a ship. Substituting the relation (3) for $\boldsymbol{n} \Phi$ and taking the $\mathrm{x}$-component of the acting force, total resistance becomes

$$
R_{t}=-\int_{A} p \cos (\boldsymbol{n}, \boldsymbol{i}) d s+x \text { component }\left[-\int_{A} \mu(\boldsymbol{n} \times \boldsymbol{\omega}) d s\right]
$$

since $q=0$ on the surface of a ship. The first integral of the right hand side of (6) is the pressure force and the second integral is the frictional force, since $\boldsymbol{n} \times \boldsymbol{\omega}$ is tangential to the surface of a ship. This shows one of the methods of separation of total resistance into its components. In this study another method of separation has been investigated which is based on the integral over the control surface $\Sigma$ enclosing a ship.

Now consider the resistance which is expressed on the control surfaces at a distance from a ship. To handle expression on the control surfaces in more usual form, the total head is introduced as follows :

$$
H=\frac{p}{\rho g}+z+\frac{1}{2 g} q^{2}
$$

Then the momentum equation (2) becomes, writing $\boldsymbol{F}=-\boldsymbol{\nabla} g \boldsymbol{z}$,

$$
-\boldsymbol{q} \times \boldsymbol{\omega}=-g \boldsymbol{\Gamma} H-\nu \Gamma \times \boldsymbol{\omega}
$$

where $\nu=\mu / \rho$ is the kinematic coefficient of viscosity, and $g$ the acceleration of gravity. For an irrotational flow

$$
\nabla H=0 \text {, }
$$


which implies that the total head has the same value throughout the fluid. For the rotational flow, on the other hand, it is assumed that the flow field can be divided into the regions inside and outside the viscous wake, and outside the viscous wake the flow is assumed to be irrotational, i.e. $H$ is constant and equals the total head of the uniform stream defined by

$$
H_{0}=\frac{p_{0}}{\rho g}+z+\frac{1}{2 g} U^{2}
$$

Inside the viscous wake, however, the total head is regarded as a function of position.

Atter some modification a more usual expression of total resistance is obtained

$$
R_{t}=\rho g \int_{\omega}\left(H_{0}-H\right) d s+\frac{\rho g}{2} \int_{S_{l}} \zeta_{w}^{2} d y+\frac{\rho}{2} \int_{S_{i}}\left[v^{2}+w^{2}-(U-u)^{2}\right] d s
$$

where $\zeta_{w}$ is the wave height at the control surface $S_{1}$, and $(u, v, w)$ are the velocity components in the direction of $x-, y$, and $z$-axes respectively as indicated in Fig. 1.

In general the total resistance expressed by (11) is not divided further, since the pressure and the velocity components must satisfy the nonlinear partial differential equation (8). In order to divide the total resistance into its components, it is necessary to introduce some approximation which is to be described in the following section.

\subsection{Linear approximation}

To divide total resistance expressed by (11) into resistance components, the well known method of approximation for viscous fluid is used, i.e. Oseen's approximation at a distance. Taking the control surfaces at a distance from a ship so that the velocity is expressed

$$
\boldsymbol{q}=U \boldsymbol{i}+\boldsymbol{q}^{\prime}
$$

where $\boldsymbol{q}^{\prime}$ is the small deviation from the uniform velocity $U i$. Then the momentum equation (8) becomes

$$
U \frac{\partial}{\partial x} \boldsymbol{q}^{\prime}=-\Gamma\left(-\frac{p}{\rho}+g z\right)-\nu \Gamma \times \omega
$$

In the viscous wake the solution of this equation (13) can be expressed as the linear combination of two kinds of velocity

$$
\boldsymbol{q}^{\prime}=\boldsymbol{q}_{r}+\Gamma \varphi
$$

where $\varphi$ is the relocity potential of irrotational motion which satisfies Laplace equation, and $\boldsymbol{q}_{r}$ is the velocity vector of rotational motion (subscript $r$ means rotational motion) and satisfies

$$
\begin{aligned}
& \left(\boldsymbol{\Gamma}^{2}-\frac{U}{\nu}-\frac{\partial}{\partial x}\right) \boldsymbol{q}_{r}=0 \\
& \Gamma \boldsymbol{q}_{r}=0
\end{aligned}
$$

Outside the viscous wake, on the other hand, since $\boldsymbol{\omega}=0$, the solution is given by

$$
\boldsymbol{q}^{\prime}=\nabla \varphi
$$

Under the linear approximation pressure is expressed by the velocity component of irrotational motion both in the viscous wake and outside the wake

$$
p=-\rho g z-\rho U-\frac{\partial \varphi}{\partial x}
$$

Now split the wave height $\zeta_{w}$ formally into the wave height due to potential motion, $\zeta_{p}$ (subscript $p$ means potential flow) and the wave height due to rotational motion, $\zeta_{r}$, i.e. $\zeta_{w}=\zeta_{p}+\zeta_{r}$. Substituting (18), (14) in the kinematical boundary condition $\boldsymbol{n q}=0$ and the dynamical boundary condition (4 b) with (3), the linearized boundary conditions on the free surface for the potential flow are given

$$
\left.\begin{array}{l}
U \frac{\partial \zeta_{p}}{\partial x}=\frac{\partial \varphi}{\partial z} \\
\zeta_{p}=-\frac{U}{g} \frac{\partial \varphi}{\partial x}
\end{array}\right\} \quad \text { for } z=0
$$

and for the rotational flow 


$$
\begin{aligned}
& U \frac{\partial \zeta_{r}}{\partial x}=w_{r} \\
& \zeta_{r}=-\frac{2 \nu}{g}\left[\frac{\partial w_{r}}{\partial z}+\frac{\partial^{2} \varphi}{\partial z^{2}}\right] \\
& \frac{\partial u_{r}}{\partial z}+2 \frac{\partial^{2} \varphi}{\partial x \partial z}+\frac{\partial u_{r}}{\partial x}=0 \\
& \text { for } z=0 \\
& \frac{\partial v_{r}}{\partial z}+2 \frac{\partial^{2} \varphi}{\partial y \partial z}+\frac{\partial w_{r}}{\partial y}=0
\end{aligned}
$$

The free surface conditions (19) for the potential flow are identical with the condition for the linearized free surface theory of inviscid fluid. Substituting the pressure and the velocity components expressed by (12), (14), (17) and (18) in (11), the total resistance becomes

$$
\begin{aligned}
R_{t}= & \frac{\rho}{2} \int_{S_{1}}\left[\left(\frac{\partial \varphi}{\partial y}\right)^{2}+\left(\frac{\partial \varphi}{\partial z}\right)^{2}-\left(\frac{\partial \varphi}{\partial x}\right)^{2}\right] d s+\frac{\rho g}{2} \int_{S_{1}} \zeta_{p}{ }^{2} d y+\frac{\rho g}{2} \int_{\omega} \zeta_{r}{ }^{2} d y \\
& +\rho g \int_{\omega} \zeta_{r} \zeta_{p} d y-2 \rho \int_{\omega} \frac{\partial \varphi}{\partial x} u_{r} d s-\rho \int_{\omega} u_{r}^{2} d s-\frac{\rho}{2} \int_{\omega}(\nabla \varphi)^{2} d s-\rho U \int_{\omega} u_{r} d s
\end{aligned}
$$

Considering the asymptotic behaviour of velocity components at a distance $X$ behind a ship, it is known that $\varphi$ has the order of $0\left(X^{-1 / 2}\right)$. By the similarity hypothesis of velocity profile in the viscous wake [8] it is assumed that $u_{r}$ is of $0\left(X^{-2 / 3}\right)$ and the sectional area of the viscous wake $\omega$ is of $0\left(X^{2 / 3}\right)$ in the three-dimensional case. As $X \rightarrow \infty$, all the terms of (21) vanish except the first, the second and the last terms. Then the total resistance is expressed asymptotically

$$
R_{t}=\frac{\rho}{2} \int_{S_{1}}\left[\left(\frac{\partial \varphi}{\partial y}\right)^{2}+\left(\frac{\partial \varphi}{\partial z}\right)^{2}-\left(\frac{\partial \varphi}{\partial x}\right)^{2}\right] d s+\frac{\rho g}{2} \int_{S_{1}} \zeta_{p}^{2} d y-\rho U \int_{\omega} u_{r} d s
$$

No term showing the interaction between rotational and irrotational velocity components appears in (22). The first and the second terms on the right hand side of (22) may be regarded as wave resistance and the third term as viscous resistance. Now let us define that wave resistance is the component of resistance attributed to the irrotational motion of fluid associated with gravity and that viscous resistance is the component of resistance attributed to the rotational motion in the viscous wake.

The total resistance can be expressed as the sum of pressure resistance and frictional resistance and at the same time as the sum of wave resistance and viscous resistance. The interaction term of both components can be neglected as a higher order component at a distance ;

$$
R_{t}=R_{p}+R_{f}=R_{w}+R_{v}
$$

where $R_{p}$ is the pressure resistance, the first integral of (6), $R_{f}$ the frictional resistance, the second integral of (6) and $R_{w}$ the wave resistance

$$
R_{w}=\frac{\rho}{2} \int_{S_{1}}\left[\left(\frac{\partial \varphi}{\partial y}\right)^{2}+\left(\frac{\partial \varphi}{\partial z}\right)^{2}-\left(\frac{\partial \varphi}{\partial x}\right)^{2}\right] d s+\frac{\rho g}{2}-\int_{S_{1}} \zeta_{p}{ }^{2} d y
$$

and $R_{i}$, the viscous resistance

$$
R_{v}=-\rho U \int_{\omega} u_{r} d s
$$

In the case of ships with small breadth/length ratio, e.g. Michell's thin ship, formal application of linear approximation gives

$$
\begin{aligned}
& R_{p} \doteqdot-2 \rho U \int_{0}^{L} d x \int_{-a}^{0} \frac{\partial \varphi}{\partial x} \frac{\partial \eta}{\partial x} d z \\
& R_{f} \doteqdot 2 \mu \int_{0}^{L} d x \int_{-a}^{0} \frac{\partial u_{r}}{\partial y} d z
\end{aligned}
$$

where $\eta$ denotes the breadth of the ship, $L$ the length and $d$ the draught. $R_{p}$ is expressed as the component of resistance due to potential motion. $R_{f}$, on the other hand, the component of resistance due to rotational motion. Therefore in this case it can be regarded that

$$
\begin{aligned}
& R_{p} \fallingdotseq R_{w} \\
& R_{f} \fallingdotseq R_{v}
\end{aligned}
$$


For ordinary ships, however, the relations $R_{p}=R_{w}$ and $R_{f}=R_{v}$ do not exist because of the nonlinear fluid motion around a ship.

\section{Method of wave analysis and wake survey}

\subsection{Method of wave analysis}

In the previous section the expression of wave resistance has been obtained in terms of velocity potential $[c f . e q .(24)]$ which satisfies the linearized free surface conditions. To determine the velocity potential from measured wave patterns, the author assumes an equivalent doublet distribution which is distributed on the keel line of a ship as a representative of a ship. Present method of wave analysis determines first the density of the doublets corresponding to the wave patterns measured along a longitudinal cut and then calculates the amplitude functions and velocity potential according to the elementary wave concept developed by Havelock [10].

Sharma's longitudinal cut method of wave analysis determines the amplitude functions directly from measured wave patterns by means of Fourier transform. In the process of Fourier transform he needs correction for the truncation of wave patterns in order to improve the accuracy of calculation of wave resistance.

In the present method of wave analysis, however, such correction for the truncation is not necessary, because the amplitude functions are not determined directly from measured wave patterns.

Assume a point doublet $M$ locating at the point $\left(x_{i}, y_{i}, z_{i}\right)$. By the linearized free surface theory the wave height $\zeta_{p}$ at a point $(x, y)$ is given

$$
\begin{aligned}
\zeta_{p}(x, y)= & -\frac{2 M}{\pi U} \int_{-\pi / 2}^{\pi / 2} d \theta \int_{0}^{\infty} \frac{n^{2}\left[n \cos n z_{i}+K_{0} \sec ^{2} \theta \sin n z_{i}\right] e^{-n|\varpi|}}{n^{2}+K_{0}^{2} \sec ^{4} \theta} d n \\
& -\frac{4 K_{0}^{2} M}{U} \int_{\alpha i}^{\pi / 2} \sec ^{4} \theta e^{K_{0} z_{i} \sec ^{2} \theta} \sin \left(K_{0} \sigma \sec ^{2} \theta\right) d \theta
\end{aligned}
$$

where

$$
\begin{aligned}
\alpha_{i} & =\tan ^{-1}\left[-\left(x-x_{i}\right) /\left(y-y_{i}\right)\right], \\
\widetilde{w} & =\left(x-x_{i}\right) \cos \theta+\left(y-y_{i}\right) \sin \theta
\end{aligned}
$$

and $K_{0}=g / U^{2}$ wave number. The first integral on the right hand side of (30) is called local wave pattern and the second integral is called free wave pattern. If it is assumed that the wave patterns of a ship can be expressed by linear combination of a limited number of wave patterns due to doublets which are distributed on the keel line from F.P. through A.P. of a ship, the wave height is given by

$$
\zeta_{p}(x, y)=\sum_{i=1}^{N} M\left(x_{i}, y_{i}, z_{i}\right) F\left(x, y ; x_{i}, y_{i}, z_{i}\right)
$$

where

$$
F\left(x, y ; x_{i}, y_{i}, z_{i}\right)=\left[\begin{array}{l}
-\frac{2}{\pi U} \int_{-\pi / 2}^{\pi / 2} d \theta \int_{0}^{\infty} \frac{n^{2}\left[n \cos n z_{i}+K_{0} \sec ^{2} \theta \sin n z_{i}\right] e^{-n \mid}}{n^{2}+K_{0}^{2} \sec ^{4} \theta} d n \\
-\frac{4 K_{0}^{2}}{U} \int_{\alpha_{i}}^{\pi / 2} \sec ^{4} \theta e^{K 0} z_{i} \sec ^{2} \theta \sin \left(K_{0} \pi \sec ^{2} \theta\right) d \theta
\end{array}\right.
$$

Since the function $F$ can be calculated previously, the density of doublets $M_{i}(i=1,2, \cdots, \mathrm{N})$ can be determined from the measured wave patterns by the method of least squares. After the density of the doublets being determined, consider the wave patterns at a great distance from a ship. As $\left(x-x_{i}\right) /\left(y-y_{i}\right) \rightarrow \infty$, the term of local wave pattern vanishes, the wave height is given asymptotically

$$
\zeta_{p}(x, y)=\sum_{i=1}^{N} M\left(x_{i}, y_{i}, z_{i}\right) F_{\infty}\left(x, y ; x_{i}, y_{i}, z_{i}\right)
$$


where

$$
F_{\infty}\left(x, y ; x_{i}, y_{i}, z_{i}\right)=-\frac{4 K_{0}^{2}}{U} \int_{-\pi / 2}^{\pi / 2} \sec ^{4} \theta e^{K_{0} \sec ^{2} z i} \sin \left(K_{0} \pi^{2} \sec ^{2} \theta\right) d \theta
$$

Changing the order of $\Sigma$ and $\int$ in (34), the wave pattern far behind a ship is expressed by

$$
\zeta_{p}(x, y)=\int_{-\pi / 2}^{\pi / 2}\left[S(\theta) \sin \left(K_{0} \sec ^{2} \theta x \cos \theta+y \sin \theta\right)-C(\theta) \cos \left(K_{0} \sec ^{2} \theta x \cos \theta+y \sin \theta\right)\right] d \theta
$$

where $S(\theta)$ and $C(\theta)$ are the amplitude functions.

$$
\left.\begin{array}{l}
S(\theta) \\
C(\theta)
\end{array}\right\}=-\frac{4 K_{0}^{2}}{U} \sum_{i=1}^{N} M\left(x_{i}, y_{i}, z_{i}\right) \sec ^{4} \theta e^{K_{0} \sec ^{2} \theta z_{i}} \sin _{\sin }^{\cos }\left(K_{0} \sec ^{2} \theta x_{i} \cos \theta+y_{i} \sin \theta\right)
$$

Under the linearized free surface conditions (19), the velocity potential which satisfies the Laplace equation can be determined by using the amplitude functions.

$$
\begin{aligned}
\varphi(x, y, z)= & U \int_{-\pi / 2}^{\pi / 2} e^{K 0 z \sec ^{2} \theta} \cos \theta\left[S ( \theta ) \operatorname { c o s } \left(K_{0} \sec ^{2} \theta \overline{x \cos \theta+y \sin \theta)}\right.\right. \\
& +C(\theta) \sin \left(K_{0} \sec ^{2} \theta \overline{x \cos \theta+y \sin \theta)]} d \theta\right.
\end{aligned}
$$

Substituting the gradient of $\varphi$ in (24), the well known expression of wave resistance is obtained

$$
R_{w}=\pi \rho U^{2} \int_{0}^{\pi / 2}\left[\{S(\theta)\}^{2}+\{C(\theta)\}^{2}\right] \cos ^{3} \theta d \theta
$$

In the routine practice wave patterns are measured by resistance type probes which are fixed transversely across the tank. Probes are usually located at 1.5 meters distance from the center line of models of 4.2 meters in length and at 3.2 meters distance for models of 7 meters in length. About 300 points of measured wave height are used for the computation. Number of doublets are 20 for models of block coefficient less than 0.60. . On the other hand for the fuller ships, i.e. $C_{b}>0.60$, 30 points of doublet are distributed not only on the keel line but on the other two lines located at a half breadth from the keel line on the bottom to obtain a good fitting of measured wave patterns.

Before the application of the present method to the measured wave patterns in practice, a numerical check of theoretical wave pattern was carried out. Fortunately Sharma presented a few theoretical wave patterns for the numerical check of methods of wave analysis[3]. One of them was used for the present numerical check. It is the theoretical wave pattern of Inuid S-201 which is generated by a continuous distribution of sources and sinks. The result of wave analysis by the present equivalent singularity method is shown in Fig. 2. The fitted wave pattern gives a good approximation of the

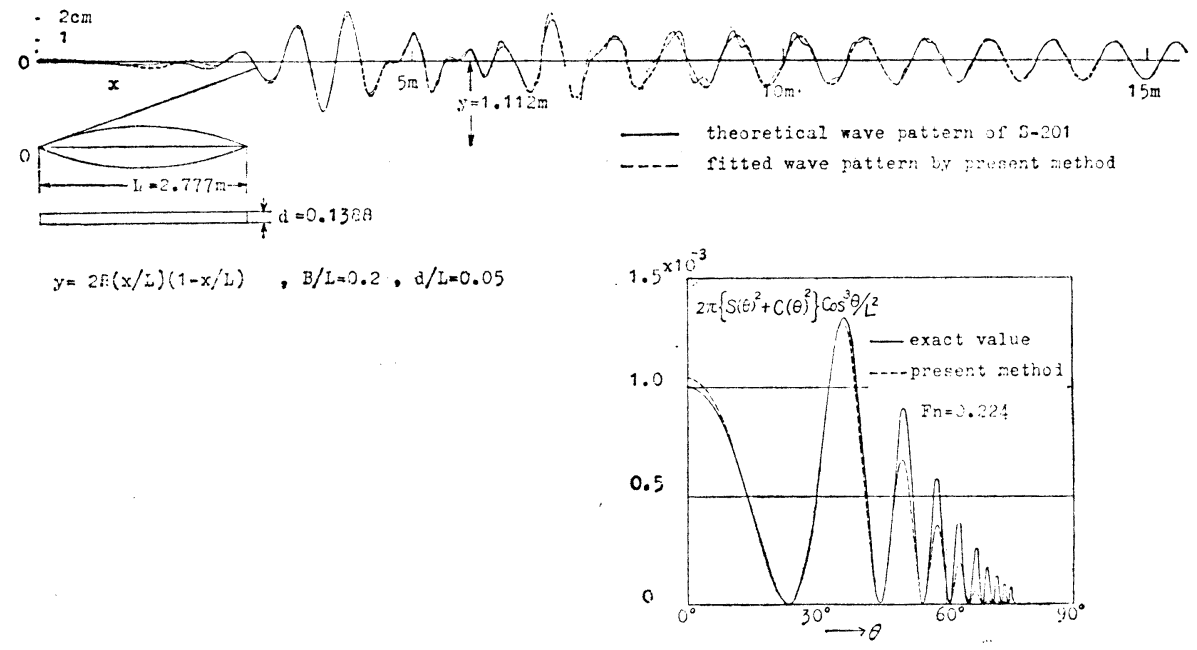

Fig. 2. Wave analysis of theoretical wave pattern 
theoretical wave pattern. The amplitude function derived by the present method is compared with the result calculated directly from sources and sinks distribution of S-201. Fair coincidence can be seen except at the range of higher value of $\theta$. The reason of the discrepancy at the higher value of $\theta$ is due to the assumption of submerged doublets for a floating ship, since amplitude functions of submerged singularities decrease exponentially at the higher value of $\theta$. This is one of the weak points of the present method. The error of calculation of wave resistance is about 8 percent. This ciegree of accuracy is probably the same order as that of Sharma's longitudinal cut method with truncation correction.

\subsection{Method of wake survey}

In the previous section the following asymptotic expression of viscous resistance was obtained

$$
R_{v}=-\rho U \int_{\omega} u_{r} d s
$$

To make this expression more practical the following relation at a distance behind a ship is used

$$
\rho g \int_{\omega}\left(H_{0}-H\right) d s \sim-\rho U \int_{\omega} u_{r} d s
$$

so as to derive viscous resistance from measurable quantities. Then an equivalent expression of (40) is

$$
R_{v}=\rho g \int_{\omega}\left(H_{0}-H\right) d s
$$

In practice, however, a control surface is chosen at a finite distance behind a ship for measuring the total head instead of the infinite distance. The position of the control surface is determined following manner. In the preliminary stage of the present study on wake survey, several control surfaces behind a ship were tried and compared. It was observed that at the nearest position $\left(0.15 L_{p p}\right.$ behind A.P.) the measured static pressure had rather strong effect due to local flow. And the control surface at $0.5 L_{p p}$ hehind A.P. of ships was selected as standard where the influence of local flow on the static pressure was hardly observed.

Under the assumption of linear treatment of flow the static pressure $p$ is expressed in terms of the velocity potential as derived in the previous section. When the static pressure is measured by manometers, the hydrostatic pressure is eliminated and eq. (18) can be rewritten as

$$
p=-\rho U \frac{\partial \varphi}{\partial x}
$$

Since the velocity potentirl $\varphi$ can be determined from measured wave patterns, numerical check was tried to see whether the static pressure measured by pitot tubes at the control surface of $0.5 L_{p p}$

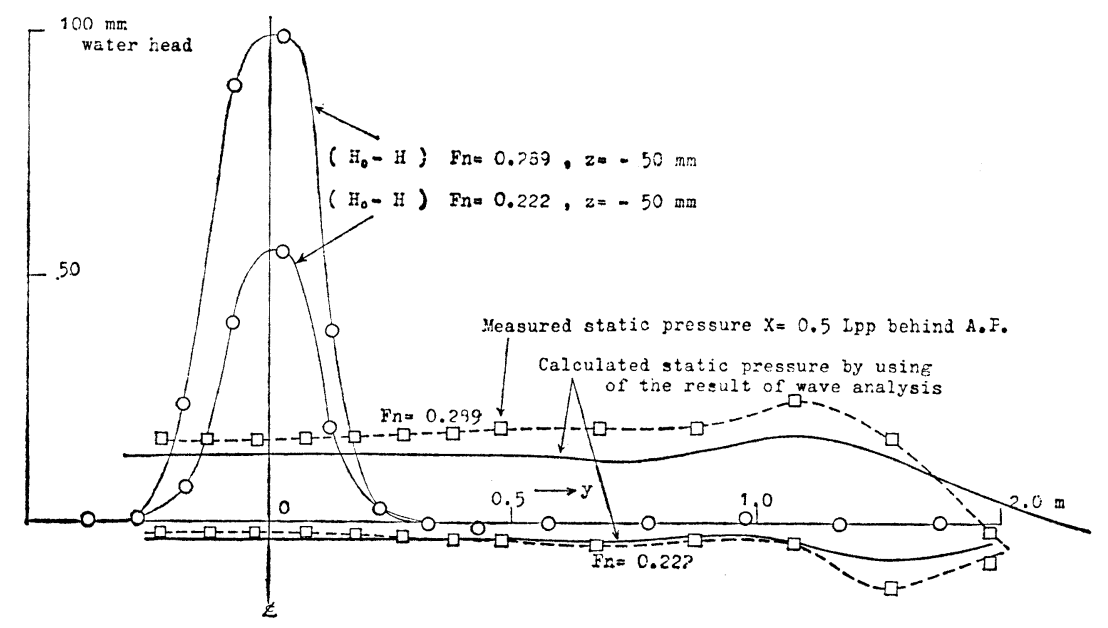

Fig. 3. Comparison of static pressure in the viscous wake (M. 1719) 
behind A.P. of ships gives a good agreement with the static pressure calculated from the measured wave patterns. Fig. 3 shows the comparison of static pressure. The calculated static pressure gives rather good approximation of the static pressure measured by pitot tubes. It is observed that the assumption of linear treatment is established successfully on the control surface.

In the routine practice of wake survey a comb type wake traverse equipment is used with 20 statichead tubes and 20 total-head tubes. The area of traverse is 3 meters in width and 1 meter in depth [6]. To determine upper boundary of the recion of viscous wake, wave heights are measured by means of lowering pointers.

\section{Experiments}

\subsection{Models used}

Both measurements of viscous resistance and wave resistance were performed on 6 models with 12 conditions in total. The particulars of these models are tabulated in Table 1.

Table 1. Particulars of models used

\begin{tabular}{|c|c|c|c|c|c|c|}
\hline \multirow{2}{*}{ Load cond. } & M. 1719 & M. 1720 & M. 1870 & M. 1870 & M. 1870 & M. 1870 \\
\hline & designed & designed & full & $1 / 2 \mathrm{DW}$ & full & $1 / 2 \mathrm{DW}$ \\
\hline$L_{p p}$ & 8.000 & 5.000 & 4.200 & 4.200 & \multirow{11}{*}{\multicolumn{2}{|c|}{ Roughened model }} \\
\hline$L_{W L}$ & 7.984 & 4.990 & 4.305 & 4.152 & & \\
\hline $\mathrm{mm}$ & 800.00 & 500.00 & 586.90 & 586.90 & & \\
\hline $\mathrm{mm}$ & 500.00 & 312.50 & 257.54 & 178.52 & & \\
\hline $\mathrm{m}^{3}$ & 1422.22 & 347.22 & 465.25 & 306.76 & & \\
\hline $\mathrm{m}^{2}$ & 9.408 & 3.675 & 3.655 & 2.964 & & \\
\hline$C_{b}$ & 0.4444 & 0.4444 & 0.7329 & 0.6971 & & \\
\hline$C_{p}$ & 0.6667 & 0.6667 & 0.7432 & 0.7113 & & \\
\hline$C_{m}$ & 0.6667 & 0.6667 & 0.9861 & 0.9799 & & \\
\hline Trim & 0 & 0 & 0 & $1 \%$ aft & & \\
\hline $\begin{array}{l}\text { Water temp. at } \\
\text { wake survey }\end{array}$ & $13.0^{\circ} \mathrm{C}$ & $15.3^{\circ} \mathrm{C}$ & $26.6^{\circ} \mathrm{C}$ & $27.2^{\circ} \mathrm{C}$ & & \\
\hline Ship form & \multicolumn{2}{|c|}{ Wigley's model } & \multicolumn{4}{|c|}{ Cargo ship model } \\
\hline \multirow{2}{*}{ Load cond. } & M. 1862 & M. 1862 & M. $1715 \mathrm{~A}$ & M. $1715 \mathrm{~A}$ & M. 1483 & M. 1483 \\
\hline & full & ballast & full & ballast & full & ballast \\
\hline$L_{p p}$ & 4.200 & 4.200 & 7.000 & 7.000 & 10.000 & 10.000 \\
\hline$L_{W L}$ & 4. 285 & 4.195 & 7.147 & 6.891 & 10.203 & 9.845 \\
\hline $\mathrm{mm}$ & 602.70 & 602.70 & 1004.52 & 1004.52 & 1435.02 & 1435.02 \\
\hline $\mathrm{mm}$ & 233.82 & 115.51 & 373.03 & 192.51 & 532.91 & 275.02 \\
\hline $\mathrm{m}^{3}$ & 452.52 & 224.39 & 2095.0 & 1038.9 & 6107.91 & 3028.72 \\
\hline $\mathrm{m}^{2}$ & 3.739 & 2.787 & 10.381 & 7.743 & 21.186 & 15.802 \\
\hline$C_{b}$ & 0.7984 & 0.7670 & & & & \\
\hline$C_{p}$ & 0.8050 & 0.7793 & & & & \\
\hline$C_{m}$ & 0.9918 & 0.9842 & & & & \\
\hline Trim & 0 & $2 \%$ aft & & & & \\
\hline $\begin{array}{l}\text { Water temp. at } \\
\text { wake survey }\end{array}$ & $27.8^{\circ} \mathrm{C}$ & $27.9^{\circ} \mathrm{C}$ & $15.0^{\circ} \mathrm{C}$ & $19.2^{\circ} \mathrm{C}$ & $24.6^{\circ} \mathrm{C}$ & $24.3^{\circ} \mathrm{C}$ \\
\hline Ship form & \multicolumn{6}{|c|}{ Geosim tanker ship model } \\
\hline
\end{tabular}

M. 1719 and M. 1720 are geosims of the Wigley's parabolic model with 8 and 5 meters in length respectively and

$$
y=2 B(x / L)(1-x / L)\left[1-(z / d)^{2}\right], L / B=10, d / L=0.0625
$$

They are similar to the model used by Lackenby and others[11] at N.P.L. in the study on ship resistance components.

M. 1870 is the model of a cargo liner of $C_{b}=0.73$ with 4.2 meters in length. This model was tested also in a roughened surface condition after the tests on smooth surface was finished, in order to obtain quantitative information on the interaction term of viscous resistance and wave resistance. 
M. 1862, M. 1715-A and M .1483 are geosims of a tanker of $C_{b}=0.80$ with $4.2,7$ and 10 meters in length respectively. Wave measurement of 10 meter model was not carried out.

\subsection{Test results}

To present the results of experiments the following dimensionless coefficients are introduced

$$
C_{t}=R_{t} / \frac{1}{2} \rho U^{2} \nabla^{2 / 3}, \quad C_{v}=R_{v} / \frac{1}{2} \rho U^{2} \nabla^{2 / 3}, \quad C_{w}=R_{w} / \frac{1}{2} \rho U^{2} \nabla^{2 / 3} \text { and } F_{n}=U / \sqrt{g L_{W L}}
$$

where $\nabla$ is the volumetric displacement of models and $L_{W L}$ the length of a water line.

Temperature correction is done on the total resistance measured by dynamometer by use of I.T.T.C. 1957 friction line so as to compare it with the sum of viscous resistance and wave resistance at the same temperature.

Since the wake surveys of models smaller than 5 meters in length are conducted at the smaller tank with 120 meters in length and 6.1 meters in width and models longer than 7 meters in length were tested at the larger tank with 165 meters in length and 12.5 meters in width, the blockage correction [9] was made on both $C_{t}$ and $C_{v}$ values. However, $C_{w}$ was not corrected for blockage effect, since all wave measurements were conducted in the larger tank.

In Fig. 4 are shown three examples of comparison of measured wave patterns and those which were approximated by the series of (32). The measured wave patterns are shown by solid lines and the fitted patterns by chain line. Fitted wave pattern of Wigley's parabolic model M. 1720 gives good approximation of measured one. At the first 4 meters of the wave pattern of a cargo ship model M. 1870 the fitted wave pattern does not give a good approximation of the measured one. A broken line in the same wave patterns shows the fitted wave pattern by distributing doublets not only on the keel line but on the other two lines on the bottom. Improvement of fitting and slight change of the amplitude function are observed. Wave pattern of a taker model M. 1862 was measured at 3 merters distance from the model center line and analysed by the three lines distribution of doublets as in the case of a cago liner model M. 1870.

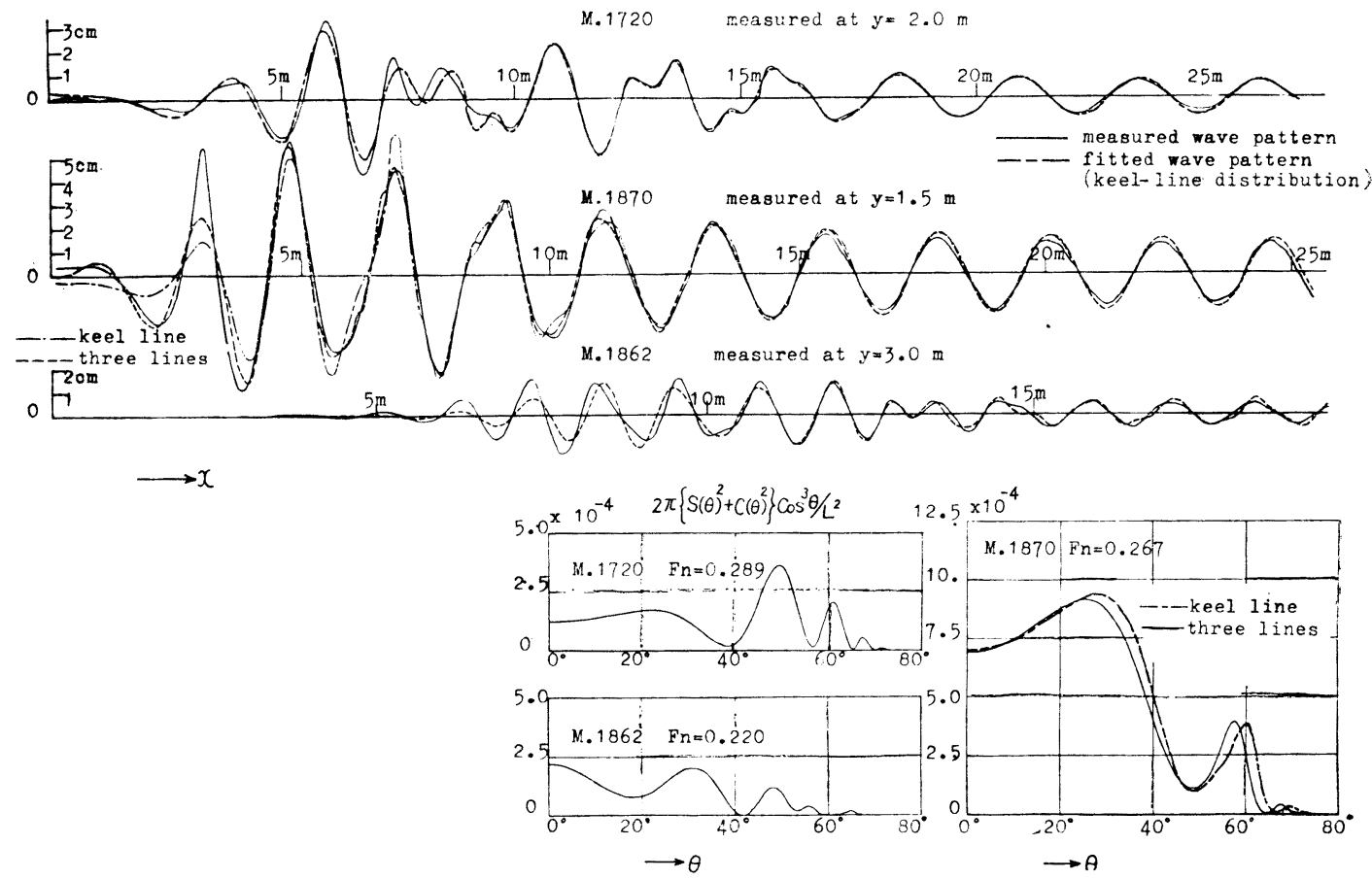

Fig. 4. Examples of wave analysis 


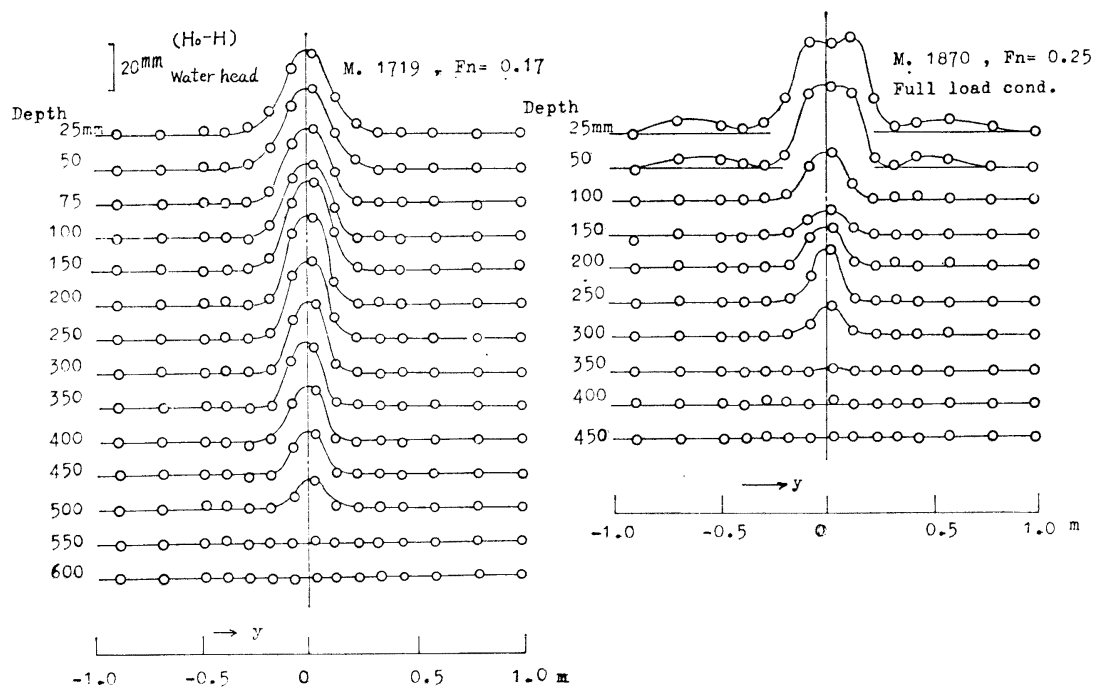

Fig. 5. Examples of wake survey

Comparison of viscous wake patterns are shown in Fig. 5. Total head loss of the Wigley's parabolic model M. 1719 shows a usual bell shape. On the other hand the total head loss of a cargo liner model M. 1870 shows a little different shape near the free surface.

Viscous resistance is derived by integrating the total head loss by planimeter. The non-dimensinal values of $C_{v}$ and $C_{w}$ are tabulated in Tables 2 through 10.

Figures 6 through 15 show the comparisons between the resistance components. Total resistance coefficient $C_{t}$ measured by resistance dynamometer is shown by solid lines and the sum of viscous resistance and wave resistance components derived by the present method is shown by black circles. It is evident from these comparisons that the sum of viscous resistance and wave resistance agrees well with the total resistance measured by dynamometer not only for the fine models (e.g. Fig. 6)

Table 2. M. 1719 wake survey and wave analysis results

\begin{tabular}{c|c|c|c}
\hline$F_{n}$ & $C_{v}$ & $F_{n}$ & $C_{w}$ \\
\hline 0.070 & 0.03307 & & \\
0.108 & 0.02448 & & \\
0.169 & 0.02447 & 0.170 & 0.00139 \\
0.222 & 0.02400 & 0.223 & 0.00311 \\
0.249 & 0.02412 & 0.250 & 0.00583 \\
0.267 & 0.02391 & 0.267 & 0.00507 \\
0.288 & 0.02459 & 0.289 & 0.00902 \\
0.319 & 0.02354 & 0.320 & 0.01240 \\
\hline
\end{tabular}

Table 3. M. 1720 wake survey and wave analysis results

\begin{tabular}{c|c|c|c}
\hline$F_{n}$ & $C_{v}$ & $F_{n}$ & $C_{w}$ \\
\hline 0.071 & 0.03165 & & \\
0.110 & 0.02838 & & \\
0.170 & 0.02770 & 0.170 & 0.00102 \\
0.222 & 0.02608 & 0.223 & 0.00309 \\
0.249 & 0.02622 & 0.250 & 0.00560 \\
0.266 & 0.02535 & 0.267 & 0.00580 \\
0.289 & 0.02501 & 0.289 & 0.00813 \\
0.321 & 0.02464 & 0.320 & 0.01043 \\
0.354 & 0.02338 & 0.354 & 0.00989 \\
0.400 & 0.02540 & 0.397 & 0.01538 \\
\hline
\end{tabular}

Table 4. M. 1870 wake survey and wave analysis results ; Full

\begin{tabular}{c|c|c|c|c}
\hline$F_{n}$ & $C_{v}$ & $F_{n}$ & $C_{w}$ & \multicolumn{1}{c|}{$C_{w}$} \\
\hline 0.077 & 0.02940 & & & $\begin{array}{l}\text { roughened } \\
\text { surface }\end{array}$ \\
0.108 & 0.02961 & & & \\
0.160 & 0.02751 & & & \\
0.200 & 0.02660 & 0.200 & 0.00056 & 0.00056 \\
0.223 & 0.02682 & 0.223 & 0.00170 & 0.00111 \\
0.249 & 0.03001 & 0.249 & 0.00388 & 0.00330 \\
0.267 & 0.03232 & 0.266 & 0.00735 & 0.00577 \\
0.290 & 0.03597 & 0.274 & 0.00925 & 0.00836 \\
& & 0.289 & 0.01944 & 0.01673 \\
\hline
\end{tabular}

Table 5. M. 1870 wake survey and wave analysis results ; $1 / 2 \mathrm{DW}$

\begin{tabular}{c|c|c|c|c}
\hline$F_{n}$ & $C_{v}$ & $F_{n}$ & $C_{w}$ & \multicolumn{1}{c}{$C_{w}$} \\
\hline 0.079 & 0.03357 & & & roughened \\
surface \\
0.110 & 0.03164 & & & \\
0.162 & 0.02618 & 0.170 & & \\
0.204 & 0.02424 & 0.204 & 0.00106 & 0.00075 \\
0.227 & 0.02519 & 0.228 & 0.00192 & 0.00166 \\
0.253 & 0.02653 & 0.255 & 0.00401 & 0.00328 \\
0.271 & 0.02816 & 0.272 & 0.00751 & 0.00634 \\
0.295 & 0.03154 & 0.280 & 0.01037 & 0.00924 \\
& & 0.295 & 0.01569 & 0.01626 \\
\hline
\end{tabular}


Table 6. M. 1862 wake survey and wave analysis results ; Full load

\begin{tabular}{c|c|c|c}
\hline$F_{n}$ & $C_{v}$ & $F_{n}$ & $C_{w}$ \\
\hline 0.130 & 0.02748 & 0.150 & 0.00017 \\
0.179 & 0.02675 & 0.173 & 0.00036 \\
0.200 & 0.02832 & 0.180 & 0.00038 \\
0.220 & 0.02955 & 0.200 & 0.00066 \\
& & 0.210 & 0.00174 \\
& & 0.220 & 0.00357
\end{tabular}

Table 7. M. 1862 wake survey and wave analysis results ; Ballast

\begin{tabular}{c|c|c|c}
\hline$F_{n}$ & $C_{v}$ & $F_{n}$ & $C_{w}$ \\
\hline 0.131 & 0.03302 & 0.183 & 0.00051 \\
0.182 & 0.03568 & 0.203 & 0.00052 \\
0.223 & 0.03843 & 0.214 & 0.00061 \\
& & 0.224 & 0.00093
\end{tabular}

Table 8. M.1715A wake survey and wave analysis results ; Full

\begin{tabular}{c|c|c|c}
\hline$F_{n}$ & $C_{v}$ & $F_{n}$ & $C_{w}$ \\
\hline 0.100 & 0.02671 & & \\
0.131 & 0.02658 & & \\
0.157 & 0.02665 & 0.155 & 0.00037 \\
0.184 & 0.02557 & 0.180 & 0.00032 \\
0.202 & 0.02738 & 0.200 & 0.00077 \\
0.212 & 0.02825 & 0.210 & 0.00159 \\
0.222 & 0.02904 & 0.220 & 0.00351 \\
\hline
\end{tabular}

Table 9. M.1715A wake survey and wave analysis results ; Ballast

\begin{tabular}{c|c|c|c}
\hline$F_{n}$ & $C_{v}$ & $F_{n}$ & $C_{w}$ \\
\hline 0.099 & 0.03024 & 0.102 & 0.00003 \\
0.129 & 0.02969 & & \\
0.157 & 0.03030 & 0.153 & 0.00009 \\
0.179 & 0.03137 & & \\
0.198 & 0.03229 & 0.203 & 0.00061 \\
0.220 & 0.03489 & 0.230 & 0.00186 \\
0.238 & 0.03631 & & \\
\hline
\end{tabular}

Table 10. M. 1483 wake survey results

\begin{tabular}{c|c|c|c}
\hline \multicolumn{2}{c|}{ Full load } & \multicolumn{2}{|c}{ Ballast load } \\
\hline$F_{n}$ & $C_{v}$ & $F_{n}$ & $C_{v}$ \\
\hline 0.130 & 0.02152 & 0.132 & 0.02825 \\
0.180 & 0.02235 & 0.132 & 0.03049 \\
0.201 & 0.02321 & 0.224 & 0.03280 \\
\hline
\end{tabular}

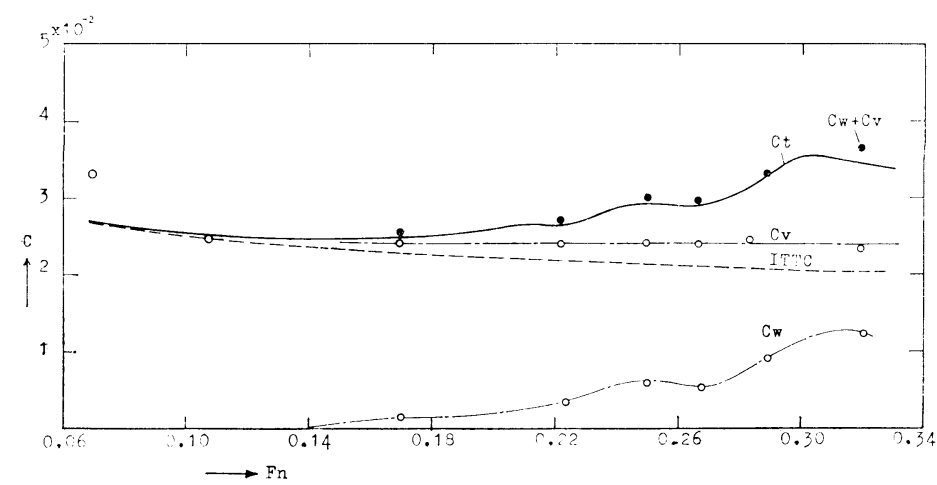

Fig. 6. Resistance components of Wigley's parabolic model $L_{p p}=8 \mathrm{~m}, C_{b}=0.44$

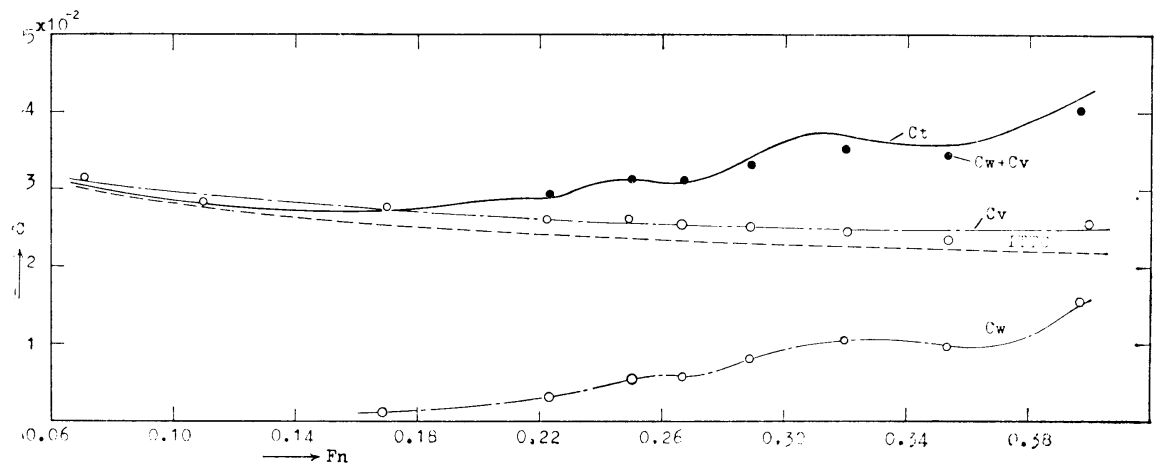

Fig. 7. Resistance components of Wigley's parabolic model $L_{p p}=5 \mathrm{~m}, C_{b}=0.44$ 


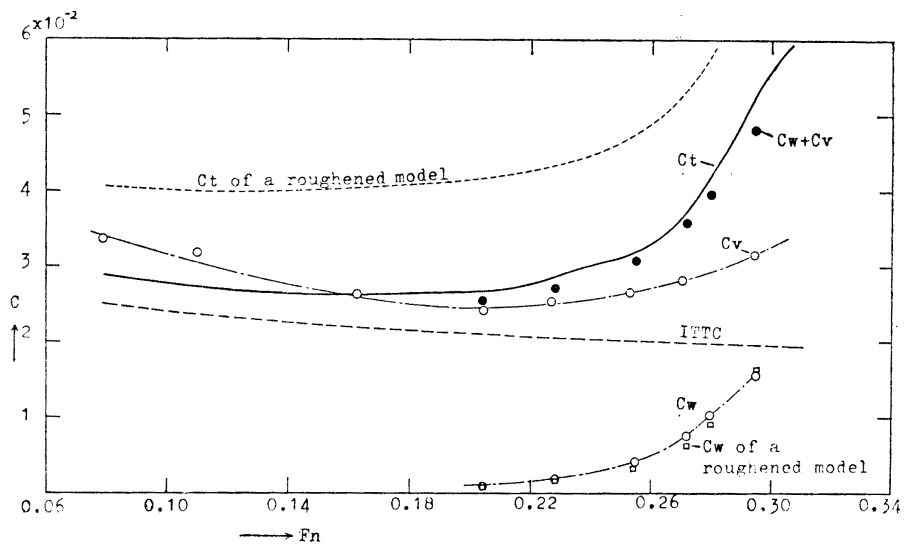

Fig. 8. Resistance components of a cargo ship model at full load condition, $L_{p p}=4.2 \mathrm{~m}, C_{b}=0.73$

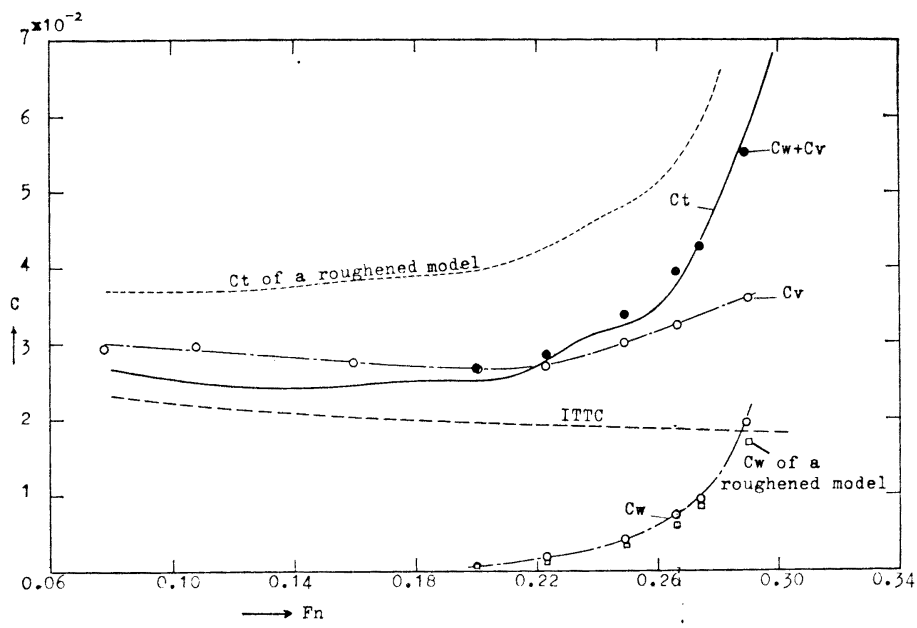

Fig. 9. Resistance components of a cargo ship model at $1 / 2 \mathrm{DW}$ load, $L_{p p}=4.2 \mathrm{~m}, C_{h}=0.70$



Fig. 10. Resistance components of a tanker ship model at full load condition, $L_{p p}=4.2 \mathrm{~m}$, $C_{b}=0.80$

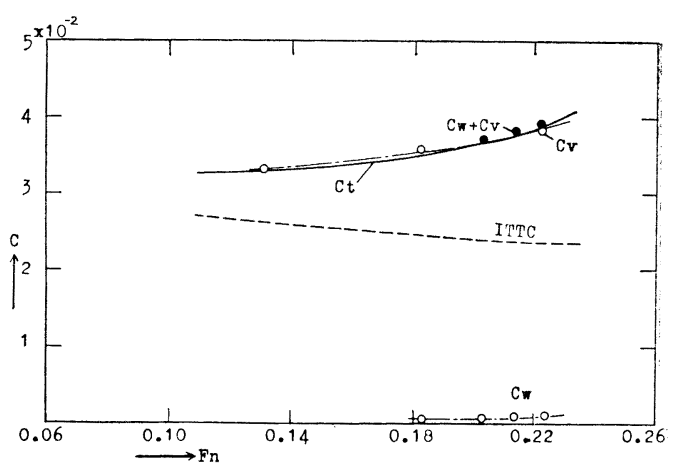

Fig. 11. Resistance components of a tanker ship, model at ballast load condition, $L_{p p}=4.2 \mathrm{~m}$, $C_{b}=0.77$ 


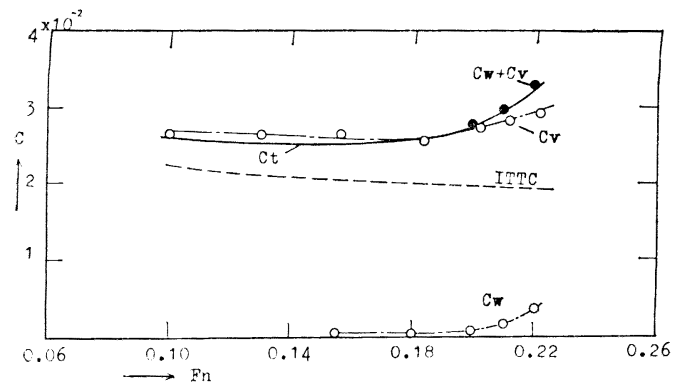

Fig. 12. Resistance components of a tanker ship model at full load condition, $L_{p p}=7 \mathrm{~m}, C_{b}=0.80$

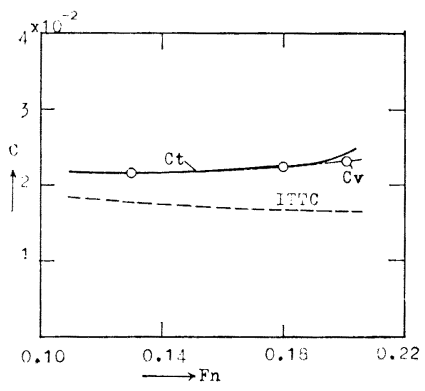

Fig. 14. Resistance components of a tanker ship model at full load condition, $L_{p p}=10 \mathrm{~m}, C_{b}=0.80$

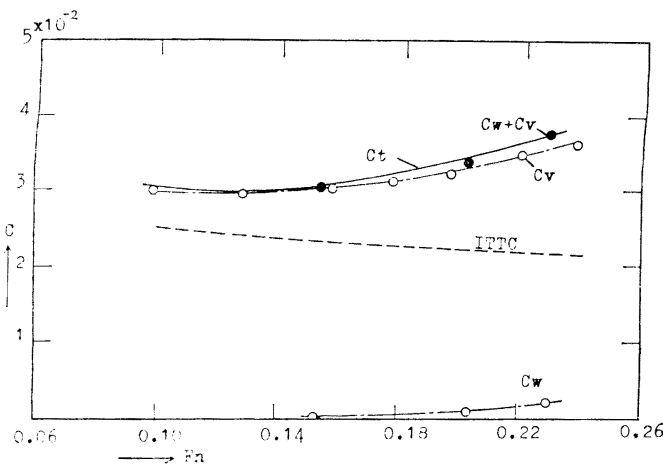

Fig. 13. Resistance components of a tanker ship model at ballast load condition, $L_{p p}=7 \mathrm{~m}, C_{b}=0.77$

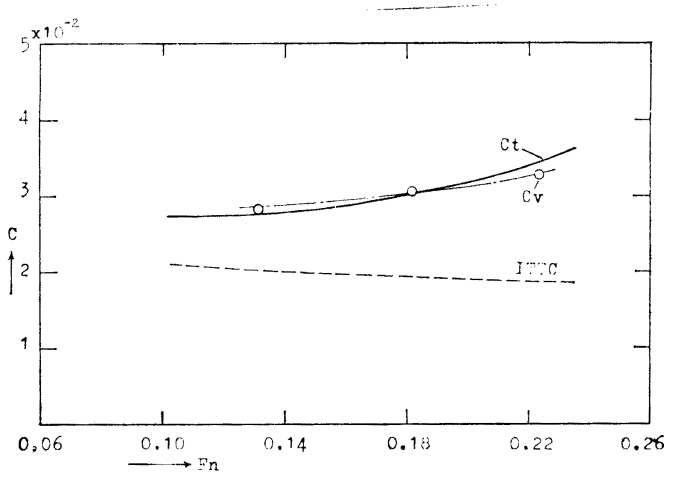

Fig. 15. Resistance components of a tanker ship model at ballast load condition, $L_{p p}=10 \mathrm{~m}, C_{b}=0.77$

but also for the fuller models (e.g. Fig. 12).

These experimental results support the validity of the theoretical analysis described in the previous section 2.2. For the Wigley's model of $C_{b}=0.44$ and $L / B=10$ the viscous resistance coefficient $C_{v}$ agrees approximately with the viscous resistance defined by means of three-dimensional extrapolation (e.g. Fig. 6 and 7). This experimental results also support the validity of the theoretical result; $C_{f} \doteqdot \mathrm{C}_{v}$ for the ship with small breadth/length ratio. On the other hand the viscous resistance coefficient curves of the fuller models (e.g. Fig. 8 or 12) have a tendency to increase in accordance with increase of wave resistance. Especially in the case of tanker models in ballast condition $C_{v}$ begins to increase at low Froude number (e.g. Fig. 13).

\subsection{Wave analysis of a roughened model}

In order to obtain quantitative information about the interaction of viscous resistance and wave resistance, wave analysis and towing tests on a roughened model were carried out using the 4.2 meter cargo liner model M. 1870 mentioned in the previous section. The whole surface of the model was roughened by scraping with a sawshape steel plate along the frame lines. The shape of the scraper is shown in Fig. 16.

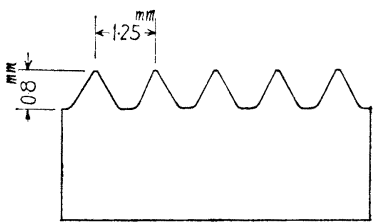

Fig. 16. Scraper for roughening a model 
As the result of roughening, total resistance derived by resistance dynamometer is increased about 50 percent as shown in Fig. 8 and 9. The wave resistance obtained by wave analysis was slightly smaller than that of a smooth model. The difference, however, is negligibly small compared with. the difference of total resistances. This implies that the effect of viscous wake on the potential flow is rather small.

Summarizing the above experimental resuits it may be concluded that the total resistance of ship. can be divided into two resistance components, i.e. viscous resistance and wave resistance and that the interaction between viscous resistance and wave resistance can be neglected as a higher order quantity. And also it may be said that the present method of theoretical analysis with a linear approximation is successfuily supported by experimental results.

\section{Conclusion}

From theoretical and experimental studies on the separation of ship total resistance into its components the following conclusions are obtained.

(1) Total resistance of ships can be divided into two components. The one is wave resistance, that is the component of resistance attributed to the potential motion of fluid associated with gravity. The other is viscous resistance that is the component of resistance attributed to the rotational motion of fluid in the viscous wake.

(2) The equivalent singularity method of wave analysis is developed for the derivation of wave resistance from the measurement of wave patterns outside the viscous wake. And a simple way of derivation of viscous resistance from the measured total head is presented.

(3) The interaction term of viscous resistance and wave resistance can be neglected as a higher order quantity.

\section{Acknowledgement}

The author wishes to express his gratitude to Dr.K. Taniguchi, Manager of Nagasaki Technical Institute, Mitsubishi Heavy Industries, Ltd. and Dr. K. Watanabe, Chief of Mitsubishi Experimental Tank in Nagasaki for their continuing guidance and encouragement.

The author also wishes to express his appreciation to all members of Mitsubishi Experimental Tank who cooperated in carrying out this investigation.

\section{References}

[1] M.P. Tulin, “The Separation of Viscous Drag and Wave Drag By Means of The Wake Survey”, DTMB Report 772, (1951)

[2] K. Eggers, “Über die Ermittludg des Wellenwiderstandes eines Shiffsmodells druch Analyse seines Wellensystems" Shiffstechnik Bd. 9-Heft 46, (1962)

[3] S.D. Sharma, "An Attempted Application of Wave Analysis Technique to Achieve Bow Wave Reduction” 6 th Naval Hydrodynamics Symposium, (1966)

[4] K.T.S. Tzou and L. Landweber, "Determination of the Viscous Drag of a Ship Model" IIHR Report No. 101, (1967)

[5] S. D. Sharma, "Zur Problematik der Aufteilung das Shiffswiderstandes in Zähigkeits und Wellenbedingte Anteile" J.S.G. 59 Band, (1965)

[6] K. Taniguchi, T. Fujita and E.Baba, "Study on the Separation of the Resistance Components" 11 th ITTC, (1966)

[ 7 ] L.M. Milne-Thomson, “Theoretical Hydrodynamics" 4 th Edition

[8] G.Birkhoff and E.H. Zarantonello, "Jets, Wakes, and Cavities" Academic Press INC., (1957)

[9] K. Taniguchi and K. Tamura, “On the Blockage Effect”, Experimental Tank (Nagasaki), Technical report No. 307, (1958)

[10] T.H.Havelock, "The Calculation of Wave Resistance” Pro. Roy. Soc. A vol. 144, (1934)

[11] H. Lackenby, "An Investigation into the Nature and Interdependence of the Components of Ship Resistance”, RINA, (1965) 